\title{
Non-invasive kinetic modelling of PET tracers with radiometabolites using a constrained simultaneous estimation method: evaluation with ${ }^{11}$ C-SB201745
}

\author{
Hasan Sari ${ }^{*}$ (D), Kjell Erlandsson ${ }^{1}$, Lisbeth Marner ${ }^{2,3}$, Ian Law ${ }^{3}$, Henrik B.W. Larsson ${ }^{3}$, \\ Kris Thielemans ${ }^{1}$, Sébastien Ourselin ${ }^{4}$, Simon Arridge ${ }^{4}$, David Atkinson ${ }^{5}$ and Brian F. Hutton ${ }^{1,6}$
}

\begin{abstract}
Background: Kinetic analysis of dynamic PET data requires an accurate knowledge of available PET tracer concentration within blood plasma over time, known as the arterial input function (AIF). The gold standard method used to measure the AIF requires serial arterial blood sampling over the course of the PET scan, which is an invasive procedure and makes this method less practical in clinical settings. Traditional image-derived methods are limited to specific tracers and are not accurate if metabolites are present in the plasma.

Results: In this work, we utilise an image-derived whole blood curve measurement to reduce the computational complexity of the simultaneous estimation method (SIME), which is capable of estimating the AIF directly from tissue time activity curves (TACS). This method was applied to data obtained from a serotonin receptor study $\left({ }^{11} \mathrm{C}-\mathrm{SB} 207145\right)$ and estimated parameter results are compared to results obtained using the original SIME and gold standard AIFs derived from arterial samples. Reproducibility of the method was assessed using test-retest data. It was shown that the incorporation of image-derived information increased the accuracy of total volume of distribution $\left(V_{T}\right)$ estimates, averaged across all regions, by $40 \%$ and non-displaceable binding potential (BP ${ }_{N D}$ ) estimates by $16 \%$ compared to the original SIME. Particular improvements were observed in $\mathrm{K}_{1}$ parameter estimates. BP $\mathrm{ND}_{\mathrm{N}}$ estimates, based on the proposed method and the gold standard arterial sample-derived AIF, were not significantly different $(P=0.7)$.

Conclusions: The results of this work indicate that the proposed method with prior AIF information obtained from a partial volume corrected image-derived whole blood curve, and modelled parent fraction, has the potential to be used as an alternative non-invasive method to perform kinetic analysis of tracers with metabolite products.
\end{abstract}

Keywords: Positron emission tomography, PET/MR, Kinetic modelling, Arterial input function

\section{Background}

Kinetic analysis methods are often used to extract quantitative measurements from positron emission tomography (PET) scans, such as cerebral blood flow, metabolism and receptor distribution. The majority of these methods require a precise knowledge of the arterial input function (AIF) to obtain the concentration of the available PET tracer in the blood plasma. However, measurement of the

\footnotetext{
*Correspondence: hasan.sari10@gmail.com; hs642@cam.ac.uk ${ }^{1}$ Institute of Nuclear Medicine, L.5 University College Hospital, 235 Euston Road, London, NW1 2BU UK
}

Full list of author information is available at the end of the article
AIF can be a challenging task in dynamic PET studies, particularly when radioactive metabolites are present in the blood. In such cases, the fraction of the metabolitefree parent compound needs to be determined before performing kinetic analysis.

The gold standard method to measure the AIF is arterial blood sampling which involves serial blood sampling from a radial artery during the course of the scan. These samples are then analysed to determine the concentration of the PET tracer in blood plasma over time. In the presence of radiometabolites, additional arterial samples 
are often required to determine the metabolite and parent compound fractions. Although it is regarded as the most accurate method for the measurement of the AIF, arterial sampling is often avoided in practice due to its invasiveness.

Image-derived input function (IDIF) estimation is often regarded as a more practical alternative to arterial sampling, where the AIF is derived from reconstructed PET images [1-3]. IDIF methods suffer from the limited resolution of PET scanners which can cause partial volume (PV) effects. Some researchers propose the use of anatomical information from MR or CT images to correct for PV effects and improve the accuracy of derived IDIFs $[4,5]$. However, IDIF methods alone are not able to separate the concentration of parent compound when radioactive metabolites are present in the blood. In such cases, several blood samples are still required to perform metabolite correction $[1,6]$.

An alternative AIF estimation method used in brain studies is the simultaneous estimation method (SIME) which is based on estimating the AIF by fitting multiple tissue time activity curves (TACs) derived from different brain regions $[7,8]$. In this approach, the AIF is defined as a parametric model where its parameters are simultaneously estimated with kinetic rate constants for brain tissues. This method was initially proposed for use in ${ }^{18} \mathrm{~F}$-FDG studies but was later shown to be applicable to other tracers [6]. Since the AIF is estimated purely computationally in SIME, this method can be particularly useful to derive the metabolite-free AIF non-invasively from the PET data. However, simultaneous estimation of AIF and kinetic rate constants from multiple regions involves estimation of a large number of parameters, resulting in bias and poor precision in the estimates [7]. Due to this problem, SIME is often complemented with several blood samples obtained at late time points to scale the estimated AIF. Furthermore, AIFs estimated using SIME tend to have accurate area under the curve but not necessarily the exact AIF shape, especially during the period following injection. Similar to conventional compartmental modelling, SIME can be more robust in deriving the macroparameters, such as volume of distribution $\left(\mathrm{V}_{\mathrm{T}}\right)$ and binding potentials (BP), rather than individual kinetic parameters [9]. This is due to macroparameters being a function of kinetic parameters which are correlated. Hence, errors on kinetic parameter estimates often cancel out in the calculation of macroparameters.

We have previously shown with simulated ${ }^{18} \mathrm{~F}$-FDG data that the performance of the SIME method can be significantly improved if some prior information about the early part of the AIF can be included from another source, such as an image-based measurement or an MRI-derived AIF [10]. In other work, prior AIF bolus information was utilised in ${ }^{18} \mathrm{~F}$-FDG studies and was shown to work well when complemented with late blood samples [11].

Another source of uncertainty in SIME is the presence of cerebral blood volume (CBV) in TACs. Although this volume is usually small in TACs extracted from brain regions, the contribution of the tracer concentration in the CBV to the total TAC might not be negligible and needs to be accounted for in the kinetic modelling. In the original SIME, the total tissue output of each region is modelled by summing the products of estimated parent AIF with the tissue and blood concentrations. This approach is not ideal since the CBV component is linked to whole blood concentration rather than the parent AIF and these concentrations can have substantial differences over the course of the scan.

In this article, we propose a constrained SIME method which utilises information from an image-derived whole blood curve [5] to reduce the complexity of the SIME. Our aim is to aid the SIME optimisation by using this image-based measurement to define the AIF peak and to differentiate whole blood activity from parent tracer activity in the plasma. Our ultimate goal is to develop a fully non-invasive method to perform kinetic analysis of PET tracers with metabolites. The method is evaluated in a study using ${ }^{11} \mathrm{C}-\mathrm{SC} 207145$ [12] which is a radioligand used in quantification of 5-HT4 receptors in serotonin studies investigating memory and learning. The performance of the constrained SIME was assessed by comparing the results to those obtained using the original SIME and the gold standard arterial blood sample-derived AIFs.

\section{Methods}

\section{SIME}

The SIME works on the assumption that the parent AIF is common to all of the TACs and can be expressed using a mathematical function. In its original implementation, Feng et al. used an input function model [13] which is a sum of a gamma variate function and two exponentials. This function has six parameters and can be written as Eq. 1.

$$
\mathrm{C}_{\mathrm{P}}(\mathrm{t})=\left(\mathrm{A}_{1} \mathrm{t}-\mathrm{A}_{2}-\mathrm{A}_{3}\right) e^{-\lambda_{1} \mathrm{t}}+\mathrm{A}_{2} e^{-\lambda_{2} \mathrm{t}}+\mathrm{A}_{3} e^{-\lambda_{3} \mathrm{t}}
$$

The six parameters of the AIF function are estimated together with the kinetic parameters by fitting the TACs for multiple brain regions simultaneously. Therefore, when a two-tissue compartment (2-TC) model with four rate constants $\left(\mathrm{K}_{1}-\mathrm{k}_{4}\right)$ is used to model $m$ number of regions of interest (ROIs), $4 m+6$ parameters are included in the optimisation. The cost function can be written as follows:

$$
\phi(p)=\sum_{i=1}^{m} \sum_{j=1}^{n} w_{i, j}\left[\bar{E}_{i}\left(\mathrm{t}_{j}\right)-M_{i}\left(\mathrm{t}_{j}\right)\right]^{2}
$$


where $\overline{\mathrm{E}}_{i}\left(\mathrm{t}_{j}\right)$ is the estimated model output, $M_{i}\left(\mathrm{t}_{j}\right)$ is the measured PET activity concentration at the $j$ th time frame of the $i$ th region. Symbol $m$ represents the number of TACs to be fitted, $n$ represents the number of time frames for each TAC and $w_{i, j}$ are the weights applied to the model and were set to 1 in our implementation to apply uniform weighting. In order to account for the measured activity in a PET scan, $\overline{\mathrm{E}}_{i}\left(\mathrm{t}_{j}\right)$ is computed by averaging $E_{i}$ over the length of the scanning interval:

$$
\begin{aligned}
& \bar{E}_{i}\left(t_{j}\right)=\frac{1}{\Delta t_{j}} \int_{t_{j}^{-}}^{t_{j}^{+}} E_{i}(s) d s \\
& E_{i}(t)=\left(1-V_{b}\right) C_{\mathrm{P}}(t) \otimes h\left[K_{1}, k_{2}, k_{3}, k_{4}\right]_{i}+V_{b} C_{\mathrm{WB}}(t)
\end{aligned}
$$

where $t_{j}^{-}=t_{j}-\Delta t_{j} / 2, t_{j}^{+}=t_{j}+\Delta t_{j} / 2$ and $\Delta t_{j}$ is length of frame $j$. $C_{\text {WB }}$ represents the whole blood TAC, $C_{\mathrm{P}}$ is the parent AIF, $h$ is the impulse response of the 2-TC model and $V_{b}$ is the cerebral blood volume. As the original SIME does not include any prior measurement of $C_{\mathrm{WB}}, C_{\mathrm{P}}$ was used to replace it in Eq. 4.

\section{Constrained SIME}

Some tracers can be converted into metabolite side products, causing an IDIF directly derived from PET images to be incorrect as the contribution of radioactive metabolites cannot be determined. The current standard method to measure the concentration of metabolites includes the collection of multiple arterial samples during the course of the PET scan. The concentration of tracer at each sample is measured to determine the whole blood curve. Next, the concentration in blood plasma is measured and plasma to whole blood ratios are plotted at each sample time point. These samples are often fitted with a linear function to be used as the plasma to whole blood ratio.

During a standard metabolite correction procedure, the fraction of parent tracer to total activity in plasma, known as the parent fraction, is determined from the plasma samples. This parent fraction is then fitted using a parametric function, such as a bi-exponential function or the Hill function, and the fitted curve is used to convert the plasma curve to a metabolite-free AIF [14]. The Hill function can be described as follows:

$$
\mathrm{f}_{\text {Hill }}(\mathrm{t})=1-\frac{(1-a) \mathrm{t}^{b}}{c+\mathrm{t}^{b}}
$$

Hence, the parent tracer concentration, $C_{\mathrm{P}}$, can be expressed as a product of the whole blood curve, fitted Hill function and plasma to whole blood ratio as shown in Eq. 6.

$$
\mathrm{C}_{\mathrm{P}}(\mathrm{t})=\mathrm{C}_{\mathrm{WB}}(\mathrm{t}) \mathrm{f}_{\text {Hill }}(\mathrm{t}) \mathrm{C}_{\text {ratio }}(\mathrm{t})
$$

where $C_{\text {ratio }}$ is the ratio of tracer concentration in plasma to whole blood as a function time and modelled using a straight line equation: $\mathrm{C}_{\text {ratio }}=d \times \mathrm{t}+e$.

If $C_{\text {WB }}$ can be reliably measured from carotid arteries in partial volume-corrected PET images, the relationship shown in Eq. 6 can be used to model the AIF in the SIME cost function. In this implementation, the input function part of the cost function contains only five unknown parameters, three from the Hill function and two from the linear function used to model the plasma to whole blood ratio. The constrained SIME method is illustrated in Fig. 1.

\section{Data acquisition}

All of the scans included in this study were performed at Copenhagen University Hospital, Rigshospitalet, Copenhagen, Denmark. The study followed the ethical guidelines from the Helsinki declaration of 1975 (revised 1983) and was approved by the Ethics Committee for Copenhagen and Frederiksberg ((KfF)01-274821). All subjects gave written informed consent. The data were collected as part of previous work published by Marner et al. [12] and have been included in the current study from the Cimbi Database [15] after approval from the Danish Data Protection Agency (2015-331-1254).

Six healthy volunteers (three males and three females, age range 21-44 years) were included in this study. Test and retest scans were performed on the same day with identical protocols. The PET data were acquired on an 18-ring GE Advance scanner (GE Healthcare, Milwaukee, WI, USA). Each subject received an intravenous administration of ${ }^{11} \mathrm{C}-\mathrm{SC} 207145$ with a mean activity of $572 \mathrm{MBq}$ (range 512-601 MBq), and high specific activity (mean $48.4 \mathrm{GBq} / \mu \mathrm{mol}$, range of $34.2-71.0 \mathrm{GBq} / \mu \mathrm{mol}$ ). Dynamic PET acquisition was started before the tracer injection and 3D PET data were acquired for $2 \mathrm{~h}$. 3D-filtered backprojection (6-mm Hann filter and $8.5-\mathrm{mm}$ axial ramp filter) was used in the image reconstruction and the PET images were reconstructed on $128 \times 128 \times 35$ volumes with a voxel size of $2.0 \mathrm{~mm} \times 2.0 \mathrm{~mm} \times 4.25 \mathrm{~mm}$. A transmission source was used to derive maps for attenuation correction [16]. PET data were corrected for randoms, scatter and deadtime. Frames were binned using the following durations: $6 \times 5 \mathrm{~s}, 10 \times 15 \mathrm{~s}, 4 \times 30 \mathrm{~s}, 5 \times 2 \mathrm{~min}$, $5 \times 5 \mathrm{~min}$ and $5 \times 10 \mathrm{~min}$. As part of the PET study, arterial blood samples were collected from the radial artery at 46 time points with 5-s intervals in the early part of the scan to get a good definition of the bolus shape. Whole blood and plasma radioactivity were measured in a well counter (COBRA 5003, Packard Instruments, Meriden, CT, USA). Additionally, six arterial samples were withdrawn at times $3.5,10,17.5,32.5,55$ and 85 mins to measure the concentration of metabolites. The parent fraction was measured using a column-switching HPLC method [17] which was previously shown to provide a 


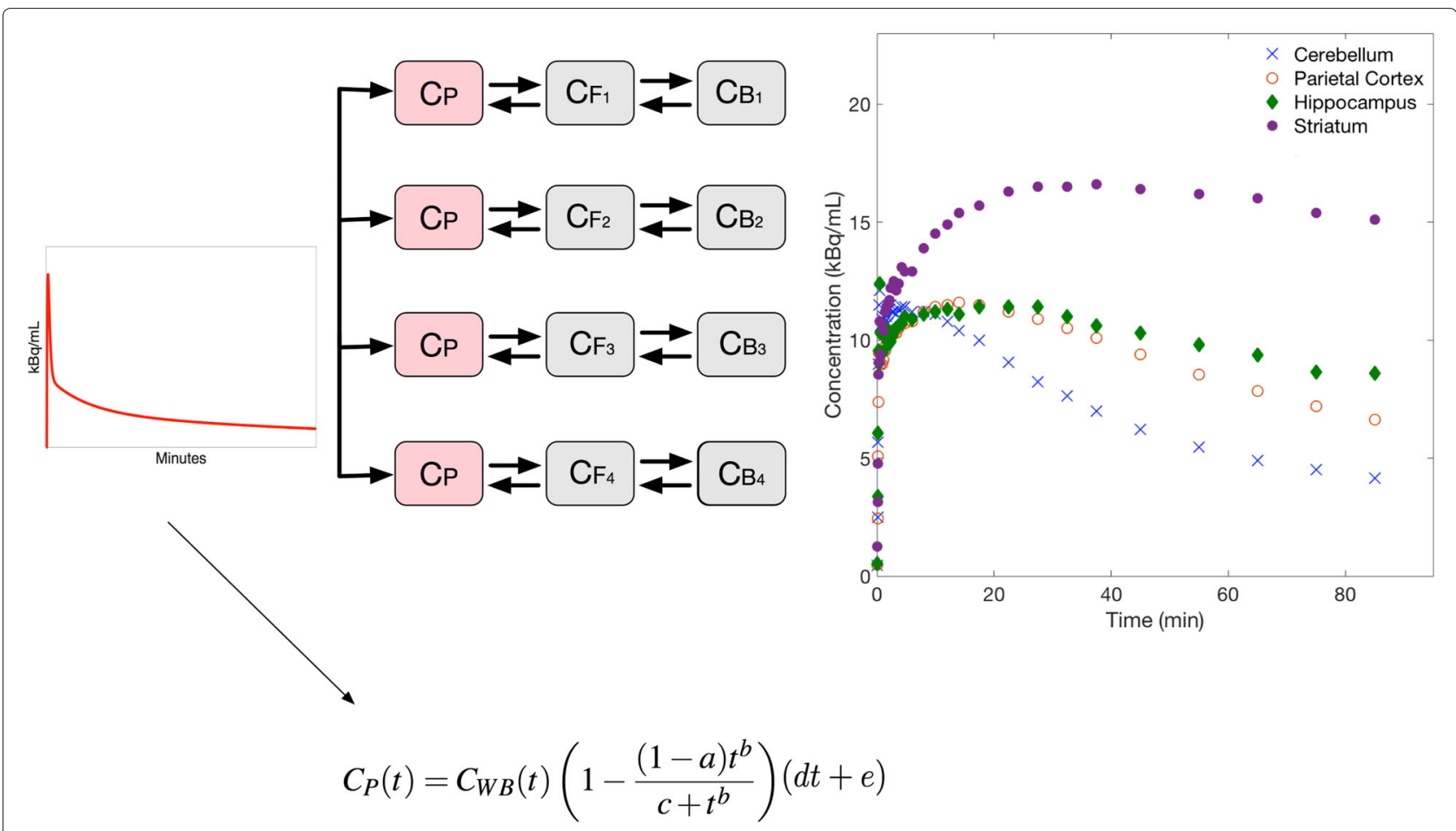

Fig. 1 Constrained SIME model used in the analysis. $C_{P}$ represents the AIF, $C_{F}$ represents the free state and $C_{B}$ of the 2-TC model. The $C_{P}$ was modelled as a product of plasma to whole blood ratio and the Hill function. Three parameters of the Hill function $(a, b, c)$ and two parameters of the straight line function $(d, e)$ were optimised simultaneously by fitting $N$ (where $N=4$ ) TACs extracted from different brain regions. $C_{W B}$ is derived from PV-corrected PET images. TACs derived from the cerebellum, parietal cortex, hippocampus and striatum from one subject are shown on the right

reliable determination of radioactive products in plasma for ${ }^{11} \mathrm{C}$-labelled radiopharmaceuticals [18]. The Hill function was fitted to the measured parent fraction and was used to obtain the metabolite-free input function. The arterial samples were corrected to account for the delay between the radial artery and the brain by applying a negative time shift to match the first PET frame. No dispersion correction was performed.

Subjects also had structural MRI scans on a 3T Siemens Trio Scanner (Siemens Healthcare, Erlangen, Germany). Three-dimensional volumetric T1-weighted MPRAGE images $(\mathrm{TR}=1380 \mathrm{~ms}, \mathrm{TI}=800 \mathrm{~ms}, \mathrm{TE}=2.6 \mathrm{~ms}$ and flip angle $=9^{\circ}$ ) of the head were acquired in sagittal planes for each subject. Images were reconstructed using a $256 \times 256 \times 256$ matrix with voxel dimensions of $1 \times 1 \times 1 \mathrm{~mm}$.

\section{Data analysis}

An adapted version of an IDIF extraction method previously validated on ${ }^{18} \mathrm{~F}$-FDG was used to derive the TAC of the tracer in the whole blood [5]. As higher resolution time-of-flight (TOF) MR angiograms were not collected as part of this study, MPRAGE images were used in the carotid artery segmentation. One of the six subjects had no visible arteries on the MPRAGE image and was excluded from the analysis as a reliable carotid artery segmentation was not feasible. For the rest of the subjects, carotid arteries were segmented using ITK-SNAP software application [19]. As part of the segmentation procedure, MPRAGE images were thresholded to retain the intensities greater than one third of the maximum image intensity. This step was used to separate the carotid arteries with higher signal from the background regions and replaced the automatic tissue classification method used in the originally published method. The tissue classification method did not yield the desired results for these datasets possibly due to the presence of high $\mathrm{T}_{1}$ signal in the background regions close to the arteries. Once the MPRAGE images were thresholded, a region-growing algorithm with active contour evolution was applied to delineate the arterial voxels [19].

The dynamic PET frames were registered to the MPRAGE images using a six-parameter rigid registration. The first 10 frames (90 s) of the dynamic PET data were added together to obtain a summed image with a high activity in the carotid arteries. This summed image was then used in the image registration which was performed using FSL Linear Image Registration Tool (FLIRT) [20]. The resulting transformation matrix was used to coalign all of the PET frames to the MPRAGE image. PET frames 
were resampled to MR space using tri-linear interpolation. The coregistered PET images were corrected for partial volume (PV) effects using the single-target correction (STC) method [5] where the segmented carotid artery mask was used as the only region of interest. An imagederived PSF of 6.8-mm full width half maximum (FWHM) was used in the PV correction. The mean intensity in the segmented artery region of each PV-corrected PET frame was calculated to derive the whole blood curve [5].

SIME requires TACs from multiple brain regions with different kinetic behaviours to estimate the AIF. In our implementation, TAC from the cerebellum was used to reflect a low binding region, TACs from the parietal cortex and hippocampus were used to reflect moderate binding regions and TACs from the striatum was used as a high binding region. Measured TACs from a single study are illustrated in Fig. 1. Segmentation of these regions was performed at Rigshospitalet, Copenhagen, Denmark [12]. The MPRAGE image of each subject was coregistered to the PET data and was parcellated into 19 different regions in the left and right hemispheres automatically using the Pvelab software package [21]. The segmented ROI masks were applied to the PET data to extract the desired TACs.

The SIME model was implemented in MATLAB (MathWorks, Inc., Natick, MA) using the COMKAT software package [22] which contains software libraries to build and solve compartmental models and perform parameter estimations. SIME optimisation was performed using the ordinary least squares method with uniform weighting applied to all time frames [23]. An analytical Jacobian of the AIF model was calculated to aid the optimisation. All four regions of interest were modelled using a two-tissue compartment model. The value of cerebral blood volume was not estimated and was assumed to be $5 \%$ for all regions. The $C_{\mathrm{WB}}$ was derived from PVcorrected PET images. Only the first $90 \mathrm{~min}$ of dynamic PET data were used in the analysis as this was previously shown to be acceptable for this tracer [12]. In the original SIME method, the six parameters of Feng's AIF model [13] are estimated together with the kinetic parameters.

In the constrained SIME method, three parameters of the Hill function and two parameters of the plasma to whole blood ratio were estimated by fitting the tissue TACs simultaneously and the estimated parameters were used with Eq. 3 to derive the parent AIF. This AIF was then used to fit each TAC individually to obtain the kinetic parameters $K_{1}, k_{2}, k_{3}$ and $k_{4}$. This second TAC fitting step was applied to re-estimate the kinetic parameters of each region of interest in a reduced parameter space per region [8]. Total volume of distribution $\left(\mathrm{V}_{\mathrm{T}}\right)$ was computed for each region using Eq. 7 and non-displaceable binding potential $\left(\mathrm{BP}_{\mathrm{ND}}\right)$ was calculated using Eq. 8, with the cerebellum used as the reference region, $\mathrm{V}_{\mathrm{ND}}$.

$$
\begin{aligned}
& \mathrm{V}_{\mathrm{T}}=\frac{K_{1}}{k_{2}}\left(1+\frac{k_{3}}{k_{4}}\right) \\
& \mathrm{BP}_{\mathrm{ND}}=\frac{\mathrm{V}_{\mathrm{T}}-\mathrm{V}_{\mathrm{ND}}}{\mathrm{V}_{\mathrm{ND}}}
\end{aligned}
$$

The results of the constrained SIME and original SIME were also compared to the gold standard method, where the metabolite-corrected AIF derived from arterial samples was used to derive the kinetic parameters, $V_{T}$ and $\mathrm{BP}_{\mathrm{ND}}$. Statistical comparisons were performed using paired $t$ tests. The results obtained from test and retest studies were used to compare the reliability of $\mathrm{V}_{\mathrm{T}}$ estimates obtained using the proposed method and arterial samples. The intraclass correlation coefficient (ICC) was computed using Eq. 9:

$$
\mathrm{ICC}=\frac{\mathrm{BMS}-\mathrm{WMS}}{\mathrm{BMS}+\mathrm{WMS}}
$$

where BMS is the mean sum of squares between subjects and WMS is the mean sum of squares between the test and retest studies for each subject. This produces a score between -1 and +1 which represents minimum and maximum reliability respectively.

\section{Results}

Throughout this section, the original SIME with no prior

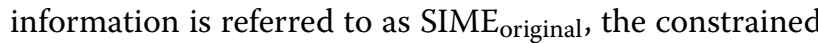
method with prior image-derived information is referred

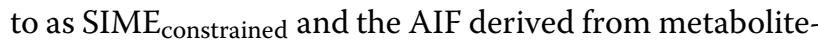
corrected arterial samples is referred to as $\mathrm{AIF}_{\text {samples }}$.

The accuracy of the image-derived whole blood TAC measurement was visually assessed against the blood sample-derived whole blood curves. Examples of these two curves are illustrated in Fig. 2 together with the

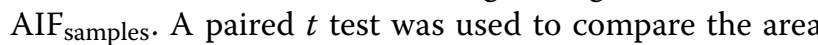
under curves (AUCs) of the measured and image-derived whole blood curves. The peak and the tail parts of the curves were assessed separately. No significant difference was observed between the AUCs of the whole blood curve tails $(P=0.10)$. However, a statistically significant difference $(P<0.05)$ was present between the imagederived and blood sample-derived whole blood peaks. A good agreement was observed in 9 of 10 subjects, but in one subject, the image-derived method yielded underestimated concentrations both in the peak and tail of the whole blood curve. The reason for this is unknown but may be caused by the errors propagated from segmentation and registration steps.

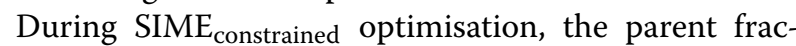
tion is estimated together with kinetic parameters by fitting the multiple tissue TACs simultaneously. Plots of simultaneously fitted TACs for a representative subject is shown in Fig. 3. The model provided good fits to the experimental data in all regions. Figure 4 illustrates the average of the estimated Hill functions using 


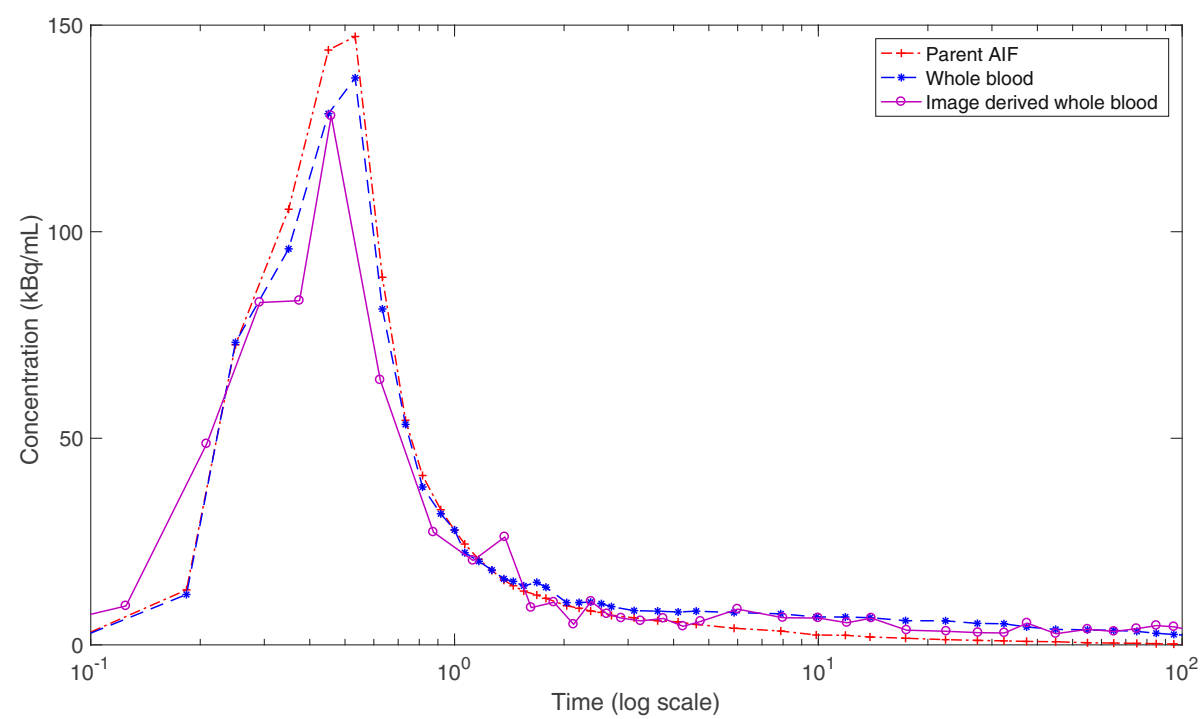

Fig. 2 Comparison of image-derived and arterial blood sample-derived whole blood curves for one subject plotted together with the metabolite-free parent AIF derived from blood samples. The $x$-axis is set to log scale to display curve peaks and tails clearly

SIME $E_{\text {constrained }}$ together with blood sample-derived parent fractions averaged across 10 datasets. Error bars are included to show variability in fitted parent fractions at blood sample data points. A very good agreement was observed between the blood sample-derived parent fractions and SIME $\mathrm{C}_{\text {constrained }}$ estimated parent fractions. Comparison of parent fraction values at metabolite sample time points did not show any significant difference between $\operatorname{SIME}_{\text {constrained }}$ and $\operatorname{AIF}_{\text {samples }}(P=0.58)$. These results indicate that $\mathrm{SIME}_{\text {constrained }}$ was able to estimate

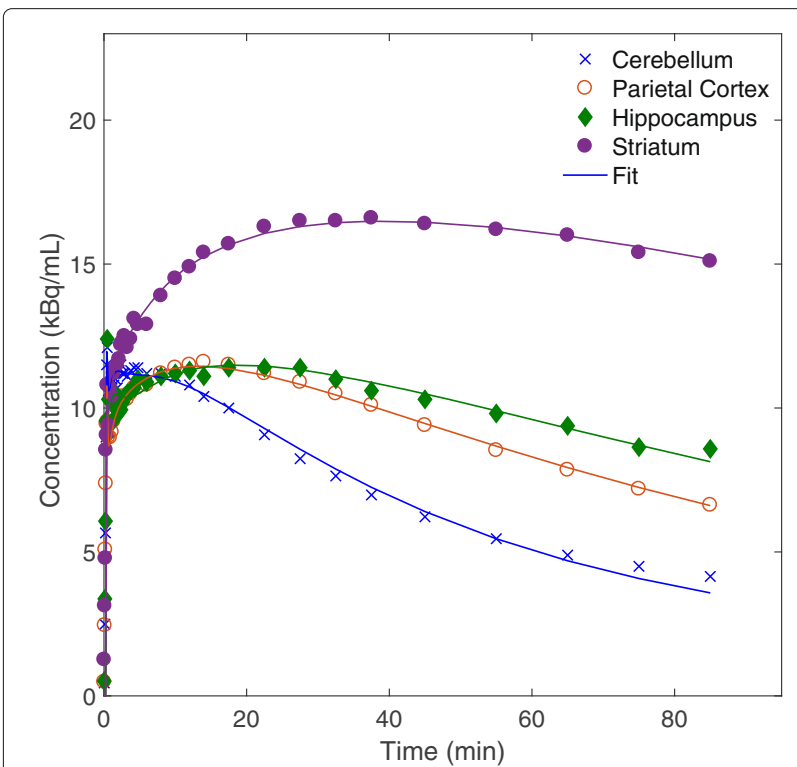

Fig. 3 TACs from one subject with simultaneously fitted curves using $\mathrm{SIME}_{\text {constrained }}$ modelling parent fractions with a comparable accuracy to blood samples. Plasma to whole blood ratio values at these time points were also compared and no significant difference was seen between the plasma to whole blood ratio curves estimated using SIME $_{\text {constrained }}$ and derived from arterial samples (paired $t$ test, $P=0.28$ ).

AIFs estimated using the three methods are plotted in Fig. 5. The AIF estimated using SIME $_{\text {constrained }}$ has a very good agreement with the gold standard $\mathrm{AIF}_{\text {samples, }}$ particularly at the AIF peak whereas SIME $\mathrm{original}_{\text {overestimated }}$ the peak. AUCs of the peak and tails of the AIFs derived using AIF $_{\text {samples }}$ and SIME $_{\text {constrained were also compared }}$ using a pairwise $t$ test and no statistically significant difference was observed ( $P=0.50$ and $P=0.52$ respectively). The estimated AIFs were used to fit TACs derived from the cerebellum, parietal cortex, hippocampus and striatum and estimated kinetic parameters were used to calculate $\mathrm{V}_{\mathrm{T}}$ in each region. The averaged results for 10 datasets are plotted in Fig. 6 together with results obtained using SIME original $_{\text {and }}$ AIF $_{\text {samples. }}$. It can be seen that using SIME $E_{\text {original yielded }} V_{T}$ estimates with $65 \%$ error for the cerebellum, $47 \%$ for the parietal cortex, $36 \%$ for the hippocampus and $50 \%$ for the striatum. Using $\operatorname{SIME}_{\text {constrained }}$ reduced the averaged percentage error to $-10 \%$ for the cerebellum, $-10 \%$ for the parietal cortex, $-7 \%$ for the hippocampus and $-11 \%$ for the striatum. Averaged across all four regions, the absolute percentage error was reduced by $40 \%$ on $\mathrm{V}_{\mathrm{T}}$ estimates and $16 \%$ in $\mathrm{BP}_{\mathrm{ND}}$ estimates. $\mathrm{SIME}_{\text {constrained }}$ underestimated the $\mathrm{V}_{\mathrm{T}}$ in all of the four regions whereas SIME produced overestimated $V_{T}$ values.

The estimated $K_{1}$ values are illustrated in Fig. 7 . SIME $E_{\text {constrained }}$ resulted in a significant improvement 


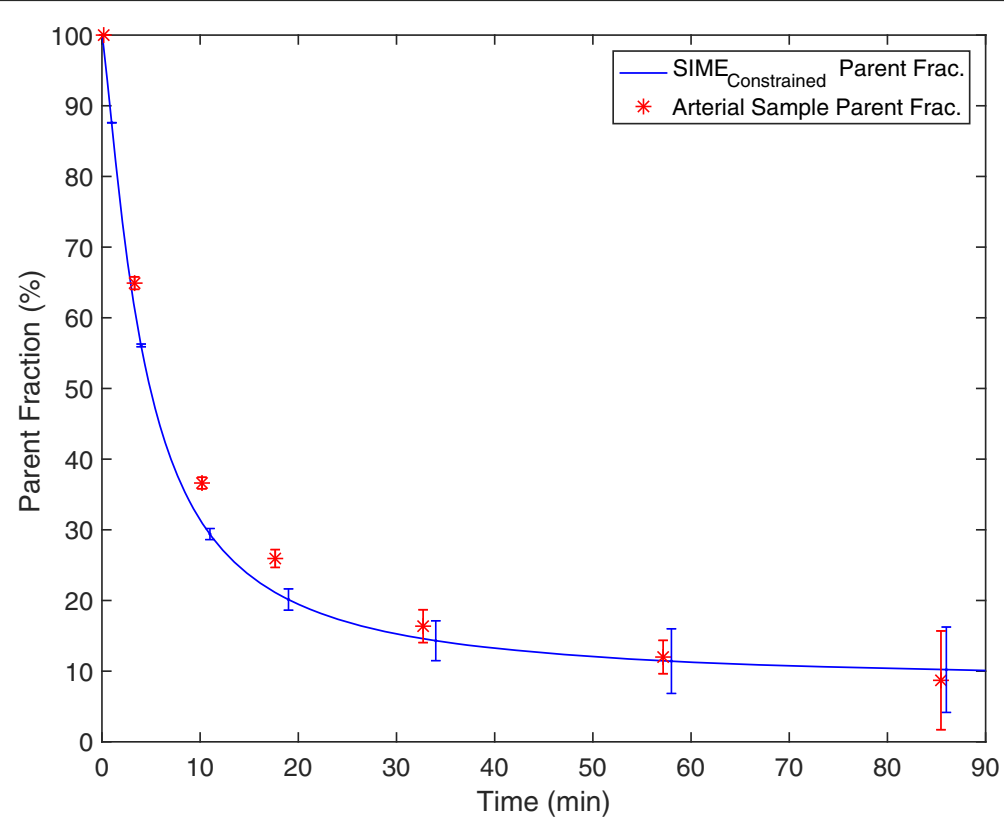

Fig. 4 Average of the parent fractions estimated using the SIME $E_{\text {constrained }}$ plotted together with average of parent fractions derived from plasma samples. Error bars represent the variation between estimated and measured parent fraction curves from 10 datasets

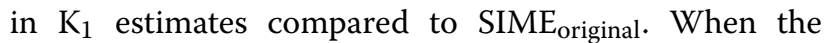
$\mathrm{SIME}_{\text {constrained }}$ was used, the percentage error dropped to $4 \%$ for the cerebellum and hippocampus, $2 \%$ for the parietal cortex and $3 \%$ for the striatum. Contrary to $\mathrm{V}_{\mathrm{T}}$ results, $\mathrm{SIME}_{\text {constrained }}$ produced slightly overestimated $\mathrm{K}_{1}$ values whereas SIME $E_{\text {original }}$ significantly overestimated $K_{1}$ in all regions. The difference between $K_{1}$ estimates between
SIME $\mathrm{E}_{\text {constrained }}$ and $\mathrm{AIF}_{\text {samples }}$ was not statistically significant $(P=0.08)$.

Non-displaceable binding potential estimates in Table 1

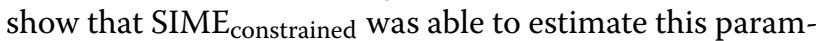
eter with a reasonable accuracy whereas larger discrepancies were observed when $\mathrm{SIME}_{\text {original }}$ was used. SIME $_{\text {original }}$ estimated $\mathrm{BP}_{\mathrm{ND}}$ with 29.7, 30.3 and $8.6 \%$ error

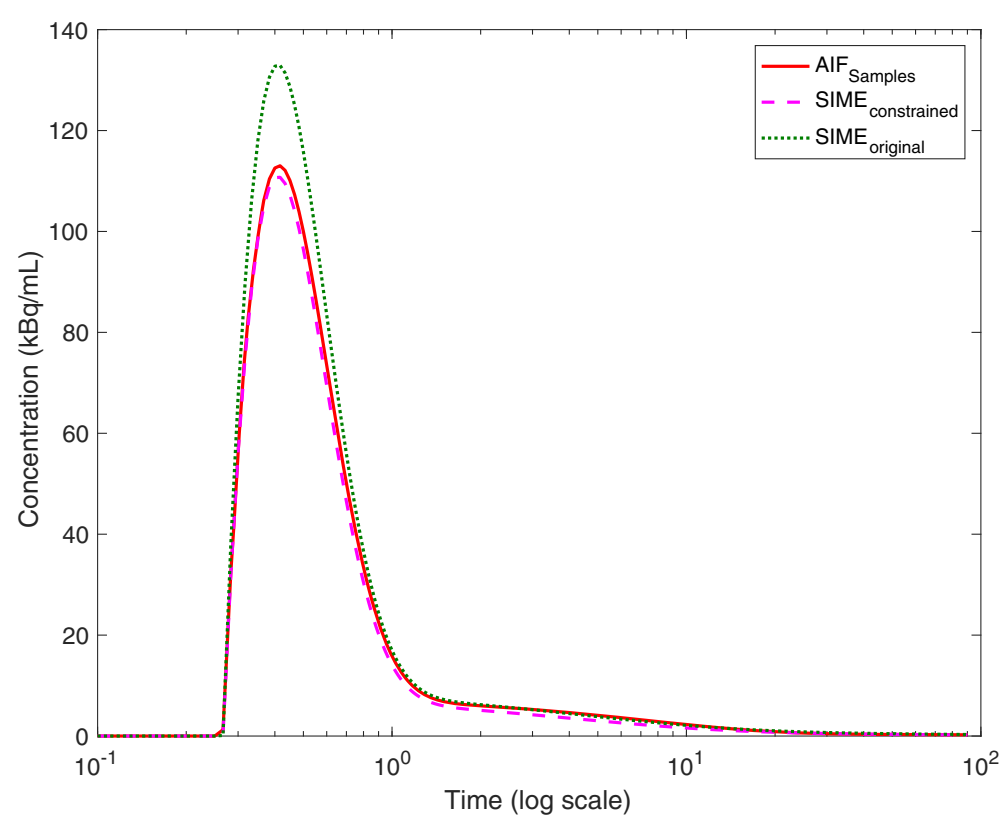

Fig. 5 AIFs derived using SIME peaks and tails clearly 


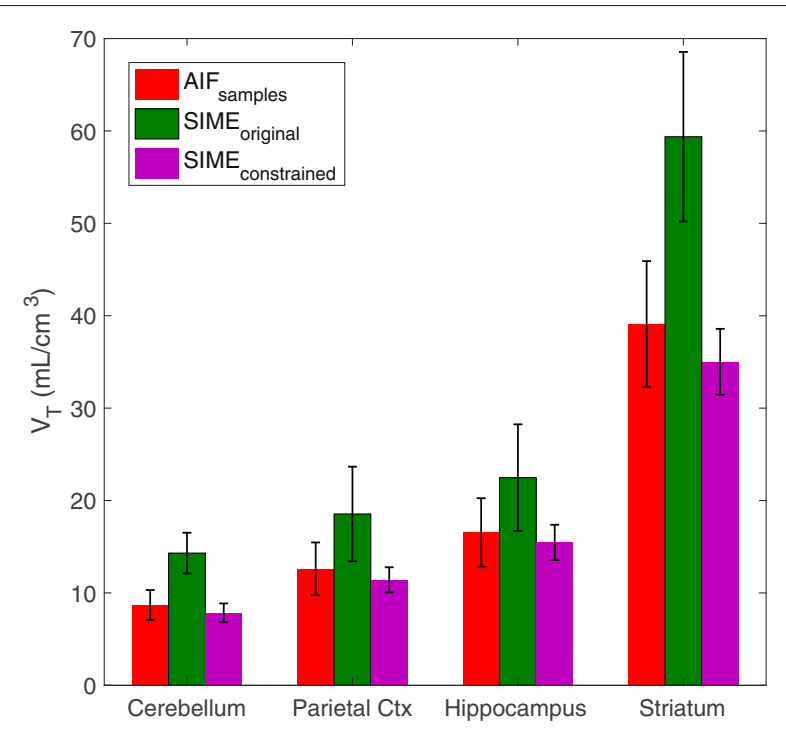

Fig. $6 V_{T}$ estimated for four brain regions using the AIFs estimated

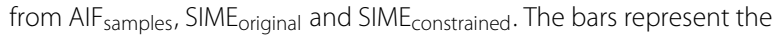
$V_{T}$ values estimated across 10 studies and error bars represent the standard deviation

for the hippocampus, parietal cortex and striatum respectively whereas these errors were reduced to 10.0, 9.0 and $1.4 \%$ respectively with $\mathrm{SIME}_{\text {constrained. However, results }}$

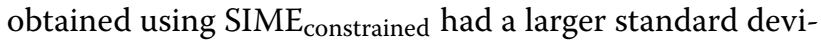
ation in $\mathrm{BP}_{\mathrm{ND}}$ estimates by $7.0 \%$ on average compared to the results obtained by arterial samples.

In Fig. 8a, $\mathrm{V}_{\mathrm{T}}$ estimations obtained using the proposed method are plotted against the ones obtained using arterial samples. While a good agreement was seen between methods at lower $V_{T}$ values, a deviation was seen as SIME $_{\text {constrained }}$ underestimated $\mathrm{V}_{\mathrm{T}}$ at higher values (i.e. in the striatum). Paired $t$ test analysis showed statistically significant difference between estimated $V_{\mathrm{T}} \mathrm{s}$ from $\mathrm{SIME}_{\text {constrained }}$ and $\operatorname{AIF}_{\text {samples }}(P<0.05)$. Figure $8 \mathrm{~b}$ shows that there was a very good relationship between the $\mathrm{BP}_{\mathrm{ND}}$ between $\mathrm{AIF}_{\text {samples }}$ and $\mathrm{SIME}_{\text {constrained. The linear fit to }}$ $\mathrm{BP}_{\mathrm{ND}}$ estimates from all brain regions and scans shows

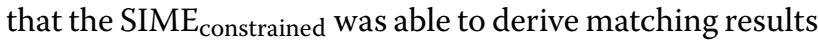
to the gold standard method, closely following the identity line. Paired $t$ test showed no statistically significant difference $(P=0.79)$ between $\mathrm{BP}_{\mathrm{ND}}$ estimates using SIME $_{\text {constrained }}$ and AIF $_{\text {samples }}$.

The intraclass correlation coefficient (ICC) results in Fig. 9 illustrate reproducibility of all three methods by comparing $\mathrm{V}_{\mathrm{T}}$ estimates obtained from test and retest

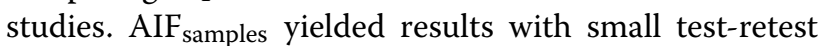
differences and high ICC, whereas SIME $E_{\text {original }}$ produced positive but much lower ICC results in all regions. $\mathrm{SIME}_{\text {constrained }}$ produced reproducible results between test and retest scans, with ICC values of 0.74 for the cerebellum, 0.84 for the parietal cortex, 0.83 for the hippocampus and 0.52 for the striatum. These results indicate that SIME $_{\text {constrained }}$ had only slightly lower reproducibility than the $\mathrm{AIF}_{\text {samples. }}$.

\section{Discussion}

In this paper, our work to incorporate image-derived whole blood concentration to improve the SIME analysis of dynamic PET data is presented. The proposed method is compared against the original SIME with no

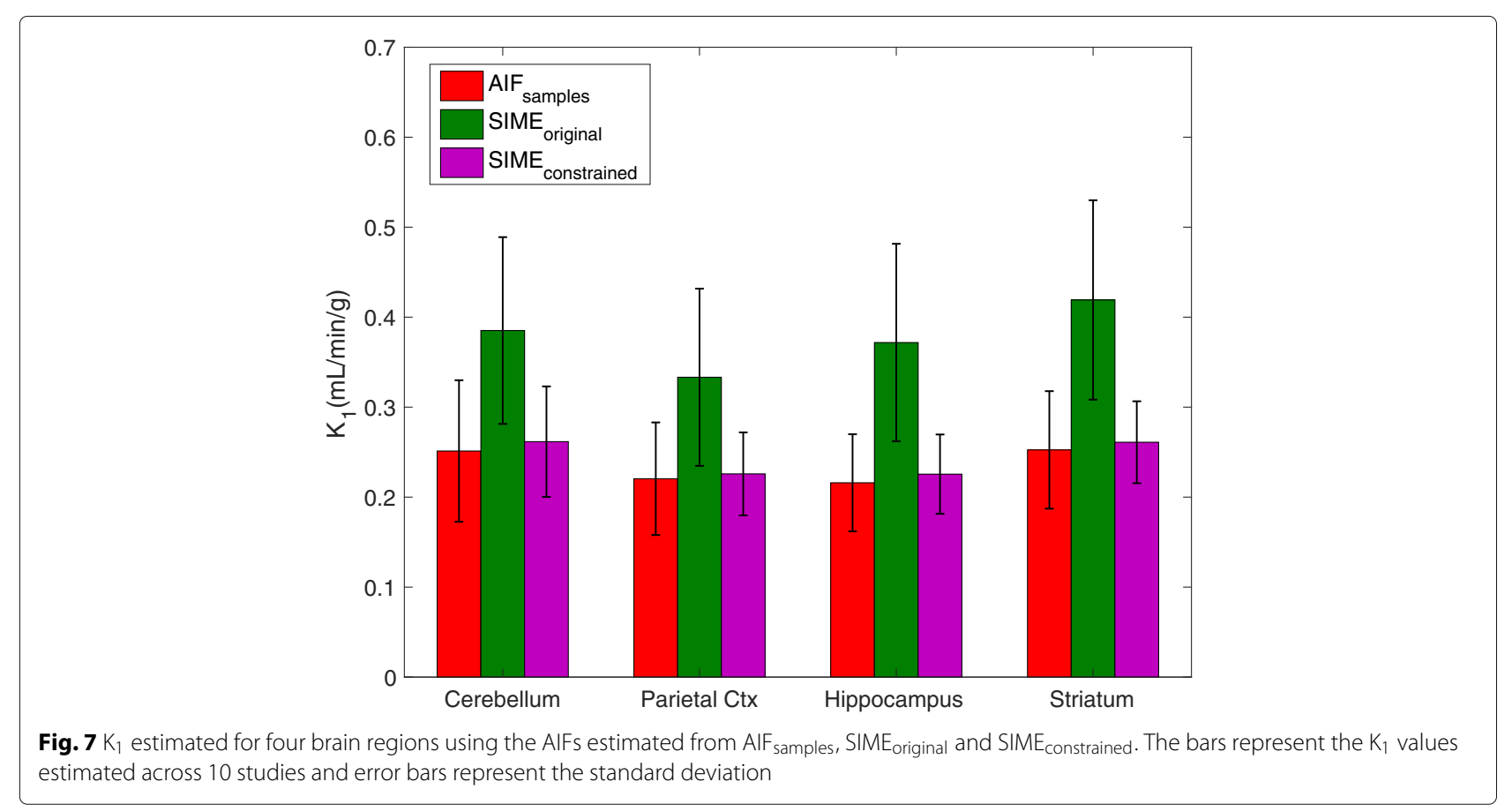


Table 1 Average non-displaceable binding potential (BP ${ }_{\mathrm{ND}}$ ) calculated across 10 scans with the standard deviation using input function derived from AlF $_{\text {samples, }}$ SIME original $_{\text {and }}$ SIME $E_{\text {constrained }}$

\begin{tabular}{llll}
\hline & AlF $_{\text {samples }}$ & SIME $_{\text {original }}$ & SIME $_{\text {constrained }}$ \\
\hline Cerebellum & - & - & - \\
Parietal ctx. & $0.42 \pm 0.08$ & $0.29 \pm 0.26$ & $0.46 \pm 0.12$ \\
Hippocampus & $0.90 \pm 0.22$ & $0.63 \pm 0.60$ & $0.98 \pm 0.23$ \\
Striatum & $3.55 \pm 0.52$ & $3.25 \pm 0.96$ & $3.50 \pm 0.57$ \\
\hline
\end{tabular}

prior information and also against the gold standard arterial sample-derived AIF. Anatomical MR images were used with the STC method to correct for partial volume effects and to obtain an accurate estimation of the whole blood TAC from PET images. This whole blood curve was included in the SIME cost function to reduce the number of estimated parameters.

The constrained SIME was used to analyse dynamic PET data from ${ }^{11}$ C-SB201745 serotonin receptor studies. Kinetic analysis of this tracer requires information about concentration of metabolite products, which is traditionally extracted from multiple arterial samples. SIME can be considered as a non-invasive alternative to analyse such tracers but large errors were observed in the estimated parameters when no prior information is included to aid AIF estimation. Using information derived from MRI-based PV-corrected PET images improved the $K_{1}$ and more importantly the $\mathrm{V}_{\mathrm{T}}$ and $\mathrm{BP}_{\mathrm{ND}}$ estimates which are the main macroparameters sought in the analysis of this tracer [12]. $\mathrm{BP}_{\mathrm{ND}}$ estimates, based on the proposed method and the gold standard arterial sample-derived AIF, were not significantly different $(P=0.7)$. The intraclass correlation results showed that the constrained SIME method was able to provide a reproducible performance in estimating $\mathrm{V}_{\mathrm{T}}$ between test and retest studies. The original SIME showed a considerably poorer performance with lower ICC scores.

The significant improvement in $K_{1}$ estimates can be explained by the direct link of this parameter to the early part of the AIF. The original SIME method has difficulty in accurately defining the AIF peaks but is more accurate in estimating the tail [6] especially when blood samples are used to anchor the estimated AIF. This makes it more successful in estimating macroparameters of kinetic analysis (i.e. $\mathrm{K}_{\mathrm{i}}, \mathrm{V}_{\mathrm{T}}$ ) rather than individual parameters. Since a reliable measurement of the whole blood curve is used in the constrained SIME, this method is superior in estimating the complete shape of the AIF curve, including the peak. This should be valid for most tracers except those with rapid uptake by red blood cells, which would result in differences between the plasma and whole blood curves.
The proposed method requires an accurate measurement of the whole blood curve from PET images, plasma to whole blood ratio and knowledge of an analytical function which can be used to define the parent fraction curve. Various models are available in the literature to model the parent fraction for different tracers [14]. In this work, the Hill function was used as it was previously shown that it is a suitable function to fit blood sample-derived parent fraction curves in ${ }^{11} \mathrm{C}-\mathrm{SB} 201745$ studies [12]. A constrained bi-exponential function, also with three parameters, is an alternative function that can be used in the analysis of this tracer, but this was not assessed in this paper. The plasma to whole blood ratio was parameterised as a straight line function where its parameters were optimised within the cost function.

In this work, the input function estimated from the constrained SIME method was used to fit individual TACs separately to estimate regional kinetic parameters. This individual TAC fitting step can be beneficial in avoiding local minima during the optimisation process, which is a risk when high number of parameters are estimated to fit multiple TACs at once. Furthermore, after estimating the AIF, it is possible to perform voxelwise kinetic analysis or fit some additional regions that were not included in the SIME procedure. In this scenario, it is best to re-fit the original SIME regions as well, so as to use the same procedure for all ROIs.

One limitation of this study was the arterial blood sampling protocol used to obtain the arterial input function. AIF was sampled in 5-10-s intervals for the first $2 \mathrm{~min}$, which might affect the accuracy of the derived AIF peak. As the blood samples are obtained from the radial artery, which is distant to the brain, the measured AIFs can suffer from delay and dispersion effects. There are available methods in the literature to correct for these effects $[24,25]$ but errors can be present even after such corrections. Therefore, it can be argued that SIME has the potential to give more specific parameter estimates as it directly measures the AIF using information from TACs in the tissue of the interest.

The constrained SIME can be used to eliminate the need for arterial sampling which is conventionally performed in the analysis of PET tracers with radiometabolite products in the blood plasma. The accuracy of the imagederived whole blood curves depends on the performance of carotid artery segmentation, PET-MR image registration and the partial volume correction of PET images. The method used to obtain the image-derived whole blood measurements was previously validated using ${ }^{18} \mathrm{~F}$-FDG datasets [5]. Results of the current study reconfirm that the blood concentration could reliably be measured from partial volume-corrected PET images. In order to use the proposed method, the study needs to include anatomical MR images to be used in carotid artery segmentation. 

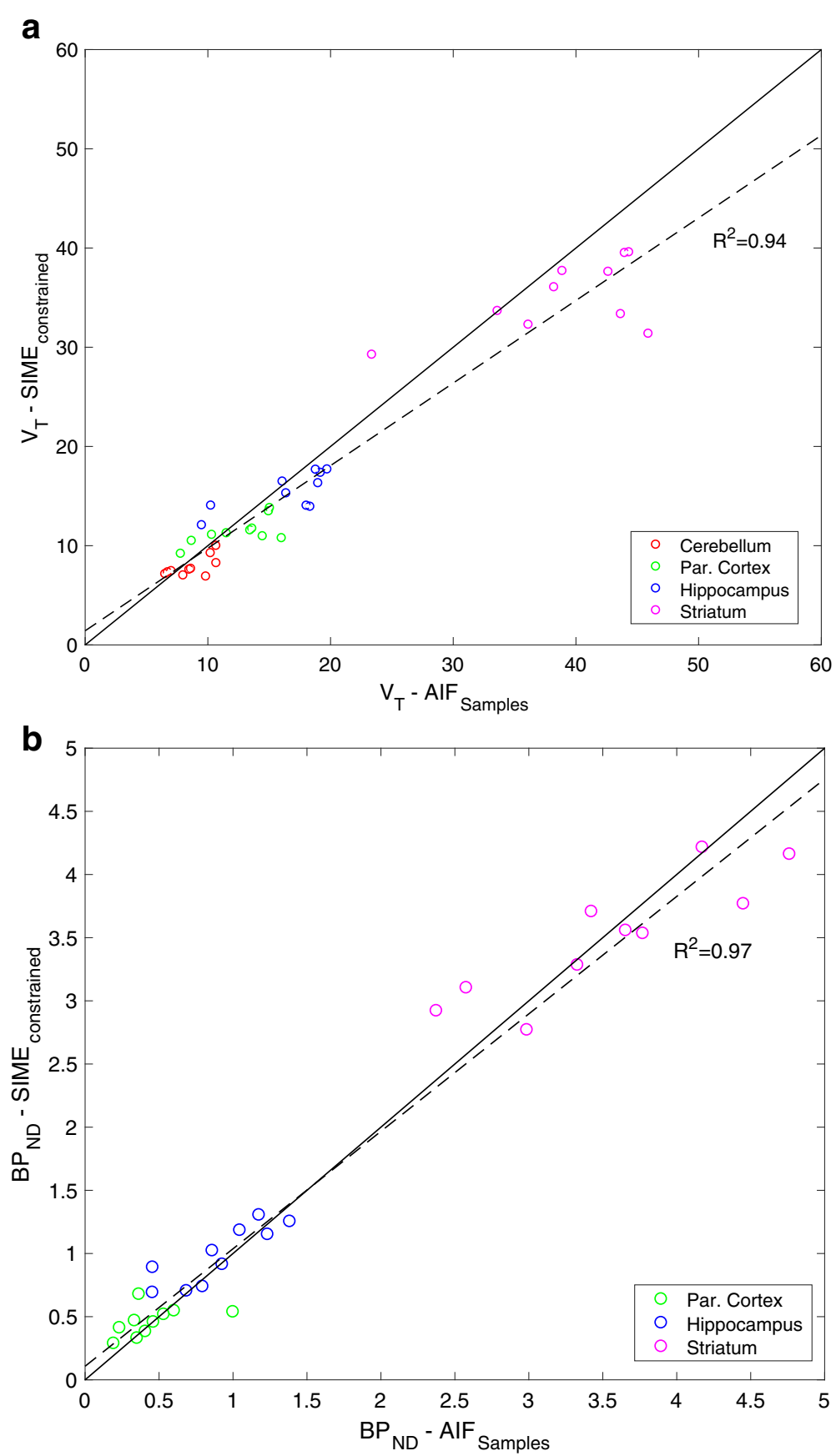

Fig. 8 a $V_{T}$ and $\mathbf{b} B P_{N D}$ estimated using SIME $E_{\text {constrained }}$ compared to $V_{T}$ and $B P_{N D}$ determined by AIF from arterial samples. Results from each scan are shown per each ROI. A linear function was fitted to the data points and plotted together with the line of identity

TOF-MR angiography is capable of providing higher resolution images with a better contrast in the arteries and therefore more straightforward artery segmentation than the T1-weighted MPRAGE images used in this study.

In this study, proof of principle of using a constrained SIME method to non-invasively analyse PET tracers with radiometabolites was demonstrated. The proposed method may be applicable to other PET tracers provided that an accurate determination of the whole blood curve can be obtained and multiple time activity curves with different kinetic behaviours are included in the field of view.

\section{Conclusions}

In this paper, we present a method which can be used to non-invasively analyse dynamic PET data in the presence 


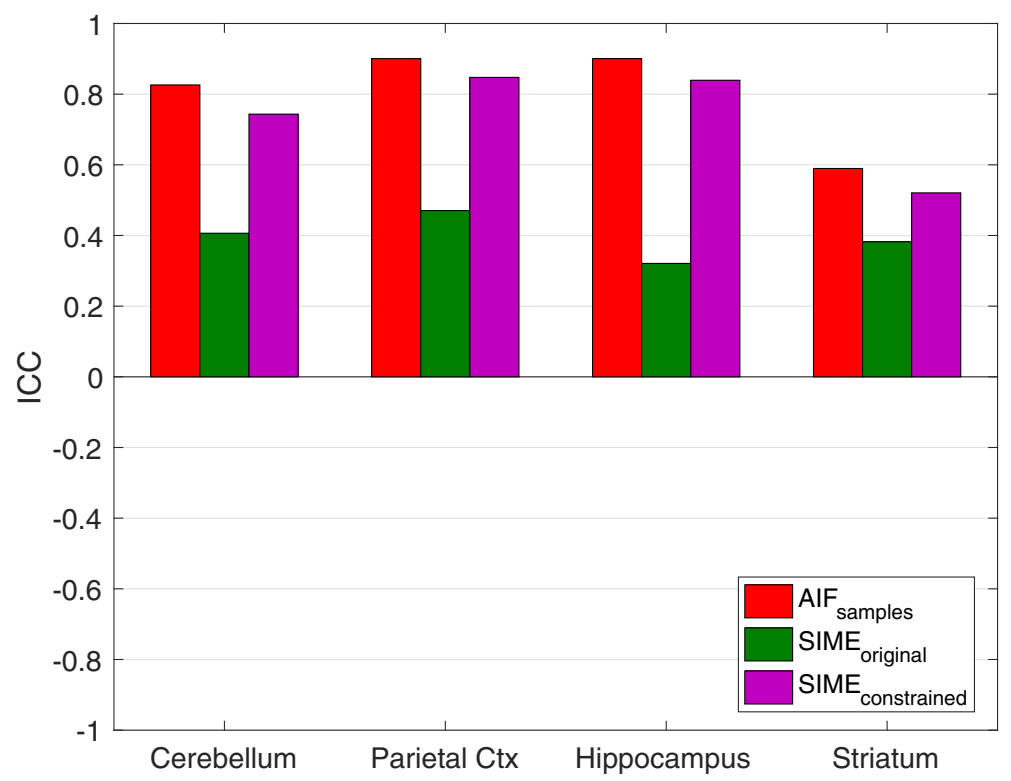

Fig. 9 Intraclass correlation coefficient (ICC) scores of the $V_{T}$ estimates obtained using AIF $_{\text {samples, }}$ SIMEoriginal and SIME maximum reliability whereas -1 represents minimum reliability

of radiometabolite side products in the plasma. The proof of principle was demonstrated with ${ }^{11} \mathrm{C}-\mathrm{SB} 201745$ data. This method is intended as a general procedure although further evaluation is required for application to other tracers.

Anatomical MR images were utilised with the STC partial volume correction technique to derive reliable estimates of the whole blood TACs from coregistered PET images. The parent fraction of the tracer in the plasma and plasma to whole blood concentration relationship were modelled using parametric equations whose parameters were estimated within the SIME optimisation. The proposed method was able to derive $K_{1}$ and macroparameters $\mathrm{V}_{\mathrm{T}}$ and $\mathrm{BP}_{\mathrm{ND}}$ with good accuracy and precision.

\section{Funding}

This work was undertaken at UCLH/UCL which receives a proportion of funding from the UK National Institute of Health Research, University College London Hospitals Biomedical Research Centres. HS was supported by an IMPACT Studentship funded jointly by Siemens and the UCL Faculty of Engineering Sciences. KE was supported by a grant from EPSRC (EP/K005278/1).

\section{Availability of data and materials}

Please contact the author for data requests.

\section{Authors' contributions}

HS contributed to the analysis and interpretation of data and drafted the manuscript. KE and BFH contributed to the interpretation of data and results. LM, IL and HBL contributed to the acquisition of data. SO, SA, KT, and DA contributed to revising the manuscript. All authors read and approved the manuscript.

\section{Ethics approval and consent to participate}

All procedures performed in studies involving human participants were in accordance with the ethical standards of the institutional and/or national research committee and with the 1964 Helsinki declaration and its later amendments or comparable ethical standards. The study was approved by the Ethics Committee for Copenhagen and Frederiksberg ((KfF)01-274821). Informed consent was obtained from all individual participants included in the study.

\section{Consent for publication}

All patients included gave written informed consent that their data could be used for scientific purposes.

\section{Competing interests}

The authors declare that they have no competing interests.

\section{Publisher's Note}

Springer Nature remains neutral with regard to jurisdictional claims in published maps and institutional affiliations.

\section{Author details}

${ }^{1}$ Institute of Nuclear Medicine, L.5 University College Hospital, 235 Euston Road, London, NW1 2BU UK. ${ }^{2}$ Neurobiology Research Unit, Center for Integrated Molecular Brain Imaging (CIMBI), Rigshospitalet, University of Copenhagen, Copenhagen, Denmark. ${ }^{3}$ Department of Clinical Physiology, Nuclear Medicine and PET, Rigshospitalet, University of Copenhagen, Copenhagen, Denmark. ${ }^{4}$ Centre for Medical Imaging Computing, Faculty of Engineering, University College London, London, UK. ${ }^{5}$ Centre for Medical Imaging, Division of Medicine, University College London, London, UK.

${ }^{6}$ Centre for Medical Radiation Physics, University of Wollongong, Wollongong, New South Wales, Australia.

Received: 1 March 2018 Accepted: 12 June 2018

Published online: 03 July 2018

\section{References}

1. Zanotti-Fregonara P, Chen K, Liow JS, Fujita M, Innis RB. Image-derived input function for brain PET studies: many challenges and few opportunities. J Cereb Blood Flow Metab. 2011;31:1986-98.

2. Zanotti-Fregonara P, Fadaili EM, Maroy R, Comtat C, Souloumiac A, Jan S, Ribeiro MJ, Gaura V, Bar-Hen A, Trébossen R. Comparison of eight methods for the estimation of the image-derived input function in 
dynamic [(18)F]-FDG PET human brain studies. J Cereb Blood Flow Metab. 2009;29(11):1825-35.

3. Bentourkia M. Determination of the input function at the entry of the tissue of interest and its impact on PET kinetic modeling parameters. Mol Imaging Biol. 2015;17(6):748-56.

4. Fung EK, Carson RE. Cerebral blood flow with [15O]water PET studies using an image-derived input function and MR-defined carotid centerlines. Phys Med Biol. 2013;58(6):1903-23.

5. Sari H, Erlandsson K, Law I, Larsson HB, Ourselin S, Arridge S, Atkinson D, Hutton BF. Estimation of an image derived input function with MR-defined carotid arteries in FDG-PET human studies using a nove partial volume correction method. J Cereb Blood Flow Metab. 2017;37(4): 1398-409.

6. Ogden RT, Zanderigo F, Choy S, Mann JJ, Parsey RV. Simultaneous estimation of input functions: an empirical study. J Cereb Blood Flow Metab. 2010;30:816-26.

7. Feng $\mathrm{D}$, Wong KP, Wu CM, Siu WC. A technique for extracting physiological parameters and the required input function simultaneously from PET image measurements: theory and simulation study. IEEE Trans Inf Technol Biomed. 1997; (4):243-54.

8. Wong KP, Feng D, Meikle SR, Fulham MJ. Simultaneous estimation of physiological parameters and the input function-in vivo PET data. IEEE Trans Inf Technol Biomed. 2001;5(1):67-76.

9. Gunn RN, Gunn SR, Cunningham VJ. Positron emission tomography compartmental models. J Cereb Blood Flow Metab. 2001;21(6):635-52.

10. Sari H, Erlandsson K, Thielemans K, Atkinson D, Ourselin S, Arridge S, Hutton BF. Incorporation of mri-aif information for improved kinetic modelling of dynamic pet data. IEEE Transactions on Nuclear Science. 2015;62(3):612-8.

11. Guo H, Renaut RA, Chen K. An input function estimation method for FDG-PET human brain studies. Nucl Med Biol. 2007;34(5):483-92.

12. Marner L, Gillings N, Comley Ra, Baaré WFC, Rabiner Ea, Wilson Aa, Houle S, Hasselbalch SG, Svarer C, Gunn RN, Laruelle M, Knudsen GM. Kinetic modeling of 11C-SB207145 binding to 5-HT4 receptors in the human brain in vivo. J Nucl Med. 2009;50(6):900-8.

13. Feng $D$, Wang $X$, Yan H. A computer simulation study on the input function sampling schedules in tracer kinetic modeling with positron emission tomography (PET). Comput Methods Programs Biomed. 1994;45(3):175-86.

14. Tonietto M, Rizzo G, Veronese M, Fujita M, Zoghbi SS, Zanotti-Fregonara P, Bertoldo A. Plasma radiometabolite correction in dynamic PET studies: insights on the available modeling approaches. J Cereb Blood Flow Metab. 2016;36:326-39.

15. Knudsen GM, Jensen PS, Erritzoe D, Baare WFC, Ettrup A, Fisher PM, Gillings N, Hansen HD, Hansen LK, Hasselbalch SG, Henningsson S, Herth MM, Holst KK, Iversen P, Kessing LV, Macoveanu J, Madsen KS, Mortensen EL, Nielsen FA, Paulson OB, Siebner HR, Stenbaek DS, Svarer C, Jernigan TL, Strother SC, Frokjaer VG. The Center for Integrated Molecular Brain Imaging (Cimbi) database. Neuroimage. 2016;124(Pt B): 1213-9.

16. DeGrado TR, Turkington TG, Williams JJ, Stearns CW, Hoffman JM, Coleman RE. Performance characteristics of a whole-body PET scanner. $J$ Nucl Med. 1994;35(8):1398-406.

17. Gillings N, Marner L, Knudsen G. A rapid, robust and fully automated method for analysis of radioactive metabolites in plasma samples from pet studies. J Labelled Comp Radiopharm. 2007;50:416.

18. Gillings N. A restricted access material for rapid analysis of [11C]-labeled radiopharmaceuticals and their metabolites in plasma. Nucl Med Biol. 2009;36(8):961-5.

19. Yushkevich PA, Piven J, Hazlett HC, Smith RG, Ho S, Gee JC, Gerig G User-guided 3D active contour segmentation of anatomical structures: significantly improved efficiency and reliability. Neuroimage. 2006;31(3): 1116-28.

20. Jenkinson M, Smith S. A global optimisation method for robust affine registration of brain images. Med Image Anal. 2001;5(2):143-56.

21. Quarantelli M, Berkouk K, Prinster A, Landeau B, Svarer C, Balkay L, Alfano B, Brunetti A, Baron JC, Salvatore M. Integrated software for the analysis of brain PET/SPECT studies with partial-volume-effect correction. J Nucl Med. 2004;45(2):192-201.

22. Muzic RF, Cornelius S. COMKAT: compartment model kinetic analysis tool. J Nucl Med. 2001;42(4):636-45.
23. Muzic RF, Christian BT, Jr RFM, Christian BT, Muzic RF. Evaluation of objective functions for estimation of kinetic parameters. Med Phys. 2006;33(2):342.

24. Meyer E. Simultaneous correction for tracer arrival delay and dispersion in CBF measurements by the $\mathrm{H} 2150$ autoradiographic method and dynamic PET. J Nucl Med. 1989;30(6):1069-78.

25. lida H, Higano S, Tomura N, Shishido F, Kanno I, Miura S, Murakami M, Takahashi K, Sasaki H, Uemura K. Evaluation of regional differences of tracer appearance time in cerebral tissues using [150] water and dynamic positron emission tomography. J Cereb Blood Flow Metab. 1988;8(2): $285-8$.

\section{Submit your manuscript to a SpringerOpen ${ }^{\circ}$ journal and benefit from:}

- Convenient online submission

- Rigorous peer review

- Open access: articles freely available online

- High visibility within the field

- Retaining the copyright to your article

Submit your next manuscript at $\gg$ springeropen.com 